\title{
Stress change before and after the 2011 M9 Tohoku-oki earthquake
}

\author{
Thorsten W. Becker* \\ Institute for Geophysics and Department of Geological Sciences \\ Jackson School of Geosciences, The University of Texas at Austin \\ Akinori Hashima \\ Earthquake Research Institute, The University of Tokyo \\ Andrew M. Freed \\ Department of Earth, Atmospheric, and Planetary Sciences \\ Purdue University \\ Hiroshi Sato \\ Earthquake Research Institute, The University of Tokyo
}

\begin{abstract}
Megathrust systems hold important clues for our understanding of longand short-term plate boundary dynamics, and the 2011 M9 Tohoku-oki earthquake provides a data-rich case in point. Here, we show that the F-net moment tensor catalog indicates systematic changes in crustal stress in the years leading up to the M9, due to the co-seismic effect, and for the last few years due to viscous relaxation. We explore the match between imaged stress change and the perturbations that are expected from 3-D, mechanical models of the visco-elastic relaxation and afterslip effects of the M9. While these models were constructed based on geodetic and structural seismology constraints alone, they match many characteristics of the seismicityinferred stress change. This provides additional confidence in the modeling approach, and new clues for our understanding of plate boundary dynamics for the Japan trench. The success of deterministic approaches for explor-
\end{abstract}

*Corresponding Address: Institute of Geophysics, 10100 Burnet Road (R2200) Austin, TX 78758-4445, USA. Phone: ++1 (512) 471-0410

Email address: twbeig. utexas. edu (Thorsten W. Becker) 
ing crustal stress change also implies that joint inversions using stress from focal mechanisms and geodetic constraints may be feasible. Such future efforts should provide key insights into time-dependent seismic hazard including earthquake triggering scenarios.

Keywords: crustal stress; megathrust; earthquake cycle; Tohoku-oki M9; visco-elastic relaxation

\section{Introduction}

Understanding the hazard posed by megathrust fault systems in subduction zones requires a comprehensive understanding of the degree to which the deformation of the crust and mantle can be modeled mechanically with deterministic models, and how much of that deformation is mapped into seismic strain release and hence fault interactions. The megathrust deformation cycle can be conceptually divided into long-term tectonic loading, co-seismic rupture, a short (few years) period of afterslip close to the fault zone, and longer term (decades) viscous relaxation within the lower crust or mantle asthenosphere (e.g. Wang et al., 2012). However, a range of intermediate time-scale phenomena that are not captured by a simple stick-slip megathrust cycle have been discovered more recently, including slow slip events and non-volcanic tremor (e.g. Peng and Gomberg, 2010; Obara and Kato, 2016).

Studying the perturbations that are induced by major subduction zone earthquakes presents an opportunity to refine our understanding of the multi-faceted plate boundary system. The major, destructive March 11, 2011 Tohoku-oki M9 event in Japan is a recent example (e.g. Simons et al., 2011). Analysis of geodetic time-series for GPS stations indicate that this earthquake was preceded by a remarkable modification of the effective plate boundary deformation starting $\sim 9$ years before the event (Suito et al., 2011; Ozawa et al., 2012; Mavrommatis et al., 2014; Yokota and Koketsu, 2015; Johnson et al., 2016; Loveless and Meade, 2016; Iinuma, 2018). This pre-M9 modification of the plate boundary system can alternatively be interpreted as an exceptionally long slow-slip event or perhaps a preparatory process related to the M9.

Given the large spatial extent of the fault plane and magnitude of coseismic slip (Figure 1), the post-seismic response is expected to occur on a length scale comparable to the upper mantle, and the good spatial coverage of geodetic constraints both on and offshore has already motivated a 
number of afterslip (e.g. Perfettini and Avouac, 2014) and visco-elastic relaxation or combined studies (e.g. Sun et al., 2014; Hu et al., 2016; Freed et al., 2017).

Here, we focus on the stress and strain-rate field for Japan as inferred from crustal earthquake moment tensors and how it has changed in the decade before and after the 2011 M9 earthquake. Major earthquakes are known to affect the seismically imaged stress field around them in ways that are accessible to continuum mechanics modeling (e.g. Hardebeck and Okada, 2018). In particular, rotations of inferred major compressive stress axes have been documented for the co-seismic effect near the fault zone of the Tohoku-oki event (Hasegawa et al., 2011; Hardebeck, 2012), and have been used in joint stress inversions (Yang et al., 2013). These studies suggested near-complete shear stress drop along the fault due to the M9, indicating $\sim 10 \mathrm{MPa}$ pre-earthquake deviatoric stress levels close to the fault interface (Hasegawa et al., 2012; Hardebeck, 2012), and closer to $\sim 50 \mathrm{MPa}$ in the upper crust (Yang et al., 2013).

Yoshida et al. (2012) studied the change in seismicity in the northern Honshu area before and right after the M9 and compared results with estimates from coseismic stress modeling. From changes in seismicity-inferred deviatoric stress patterns, the authors inferred that pre-M9 stress levels were regionally variable, and a triggering scenario of co-seismic stress change implied shear stress levels lower than $\sim 1 \mathrm{MPa}$ regionally before the earthquake. This is a low value, but of the same order of magnitude as estimates from strike-slip faults which suggest background stresses of $\sim 65 \%$ the coseismic stress drop (Hardebeck and Hauksson, 2001).

Here, we focus on the temporal evolution of crustal stress in a larger region around the megathrust and compare inferred temporal change before and after the co-seismic effect with that predicted from our earlier viscoelastic relaxation model (Freed et al., 2017). This provides an independent test of the mechanical relaxation model, and puts the local, co-seismic stress change into a more comprehensive context.

\section{Methods}

\subsection{Catalog analysis}

We base our crustal stress analysis on the National Research Institute for Earth Science and Disaster Resilience (NIED) F-net moment tensor catalog (Okada et al., 2004). The catalog is complete with Gutenberg-Richter, frequency-magnitude distribution $b$ value of $\approx 0.98$ down to $M_{w} \sim 4$ for 


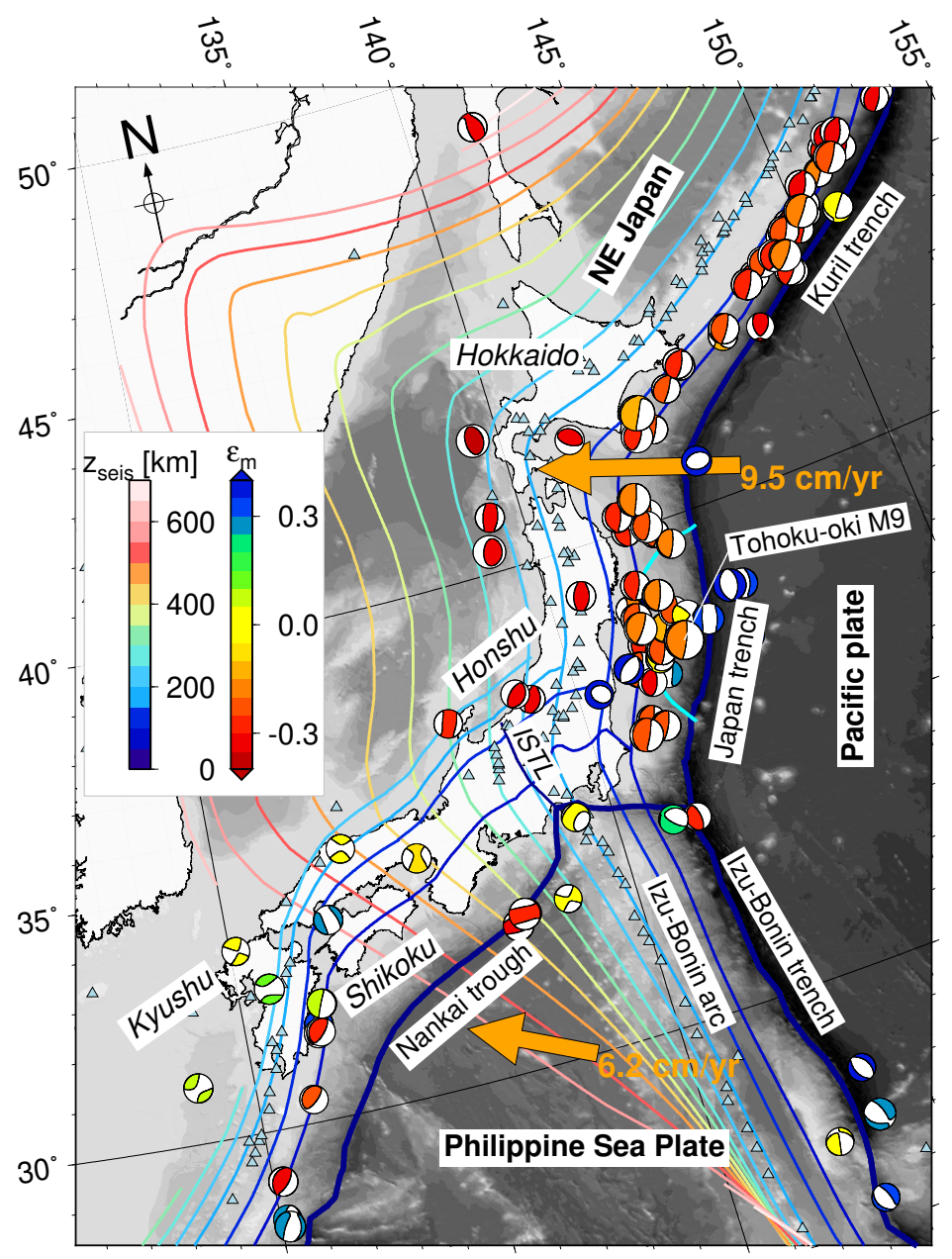

Figure 1: Seismotectonics of Japan. Moment tensors are gCMT solutions with $M_{w} \geq 6.5$ and centroid depth $\leq 50 \mathrm{~km}$ (catalog 1976 - 01/2018; Ekström et al., 2012), scaled by magnitude, and colored by the normalized horizontal strain, $\varepsilon_{m}$ (eq. 1), with blue and red indicating extensional and compressional strain, respectively. Contours are inferred top of intraslab seismicity from Gudmundsson and Sambridge (1998) and colored by depth, for $z_{\text {seis }} \geq$ $50 \mathrm{~km}$. The $M_{w}=9.1$ Tohoku-oki 2011 event and selected geographic features are labeled, ISTL: Itoigawa-Shizuoka Tectonic Line. Heavy cyan line close to the Japan trench shows the $\geq 5 \mathrm{~m}$ co-seismic slip area of the M9 from Hashima et al. (2016) for reference. Plate motions (orange vectors) are from Argus et al. (2011), with respect to the Amur plate, and plate boundaries (heavy blue lines) for the Pacific and Philippine Sea plate from Bird (2003). Light blue triangles show Holocene volcanoes from the compilation of Siebert and Simkin (2002-). 
our region of interest (Figure 2), but we allow for events $M_{w} \geq 3$ for increased coverage while maintaining relatively robust moment tensor solutions. Our basic data consist of this catalog from 1997 through 10/2017, from which we use centroid depths and moment tensor inferred magnitudes. (The Tohoku-oki 2011 event is here referred to as the "M9", but it is $M_{w} \approx 9.1$ in the gCMT (Ekström et al., 2012) and $M_{w} \approx 8.7$ in the F-net catalogs, respectively.)

We seek to primarily analyze the depth-integrated response of the crust and treat all earthquakes as being representative of a single layer, which we define as consisting of all events shallower than $36 \mathrm{~km}$ depth. This value is intended to capture the entire crust on the overriding plate on land, but will extend into the mantle and slightly into the subducting plate in oceanic domains. Using the original F-net centroid depths, the distribution of catalog events in our study region peaks at $\sim 15 \mathrm{~km}$ between 0 and $36 \mathrm{~km}$ depth, with median depths between $\sim 10$ and $15 \mathrm{~km}$ on land, and $\sim 15$ to $25 \mathrm{~km}$ in oceanic domains (Figure 2).

Fairly good depth resolution is possible within the trench regions (e.g. Hasegawa et al., 2011; Hardebeck, 2012). However, centroid depths are relatively uncertain in general, and inferred depths from the F-net catalog may at least regionally have some bias, e.g. due to 3-D velocity structure (e.g. Nakamura et al., 2016; Takemura et al., 2016). We therefore assume that a layer average provides meaningful information in lieu of more detailed depth analysis, comment on some depth-dependence below, and furthermore assume that any mislocation bias in the catalog will be temporally stationary.

Individual focal mechanisms provide direct information on the strain released in the co-seismic deformation (Kostrov, 1974), but the interpretation in terms of their relationship to the stresses driving the faulting depends on assumptions about frictional behavior and/or mechanical anisotropy (e.g. McKenzie, 1969; Twiss and Unruh, 1998; Hardebeck, 2006). We therefore consider two ways of inferring the normalized stress state, with no amplitude information included in either:

1. We infer "stress" by means of a normalized Kostrov (1974) summation which assumes that the average strain(-rate) tensor from binning of normalized moment tensors (per arbitrary unit time), irrespective of their scalar moment, is aligned with the stress tensor isotropically (e.g. Platt et al., 2008; Bailey et al., 2009).

2. We use a Michael (1984) inversion for the stress state that best fits the assumption that slip of all events considered was in the direction of 


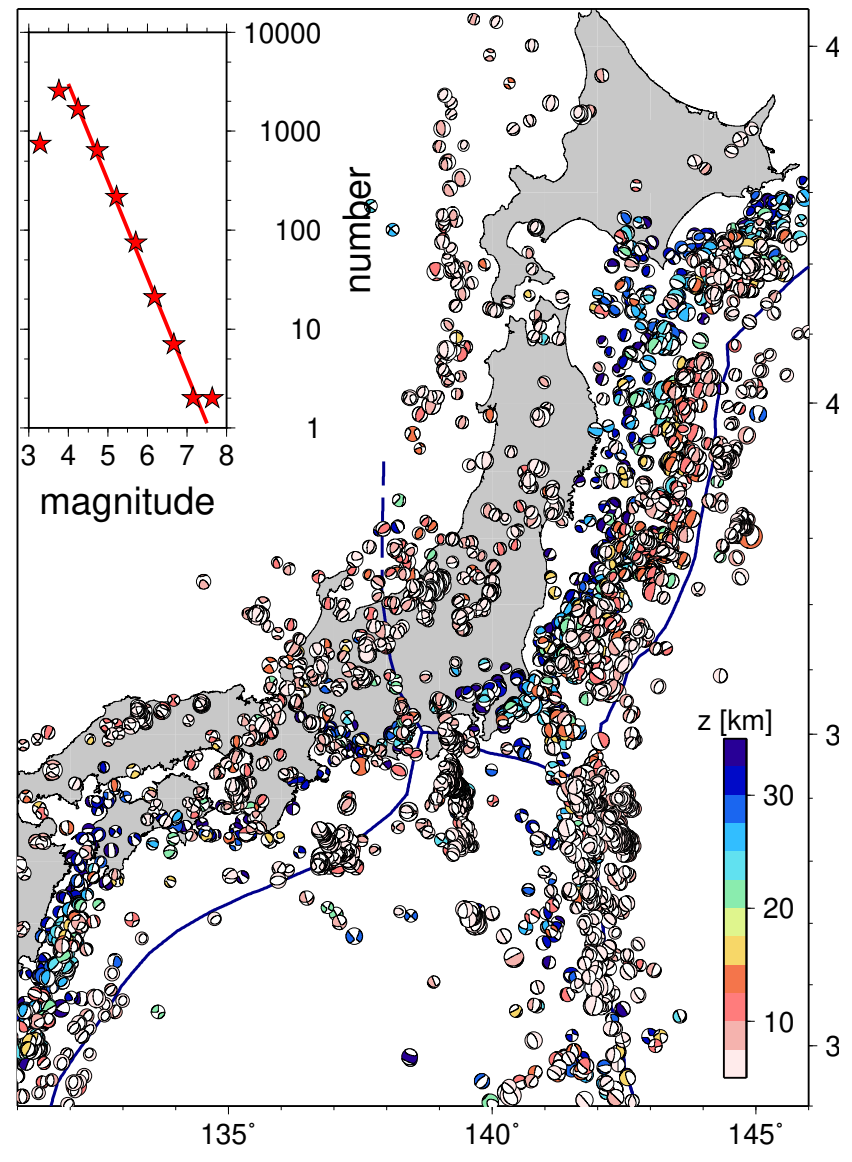

Figure 2: Shallow F-net moment tensors (Okada et al., 2004) for the study region through $t_{e}=2007$ with $M_{w} \geq 3$ and centroid depth $z \leq 36 \mathrm{~km}$ (colored) as used for the longterm stress estimate of Figure 3. Dashed blue line shows the offshore continuation of the Itoigawa-Shizuoka Tectonic Line (Figure 1) used for subsetting seismicity. Inset figure shows a binned frequency-magnitude distribution of the selected events with red line denoting the estimated $b$-value (slope) of 0.98 . 
maximum shear stress in a given fault plane. For this approach we use the two fault planes of the best-fitting double couple component of the moment tensors, applying a Monte Carlo method to randomly sample 5,000 different possible fault plane combinations without any tectonic prior information (Michael, 1987) to estimate the mean tensor and uncertainties.

Becker et al. (2005) showed that Kostrov summations predict very similar principal axes alignment compared to a spatially-smoothed stress inversion (Hardebeck and Michael, 2006) for southern California. While we will focus on the Michael (1984) type stress inversions, we consider it instructive to also compare with the simpler Kostrov summations here. Overall results are consistent, but temporal trends appear more stable in Kostrov summations, as discussed below.

We use binning of the F-net moment tensors on a regular grid in longitude and latitude at spacing $\Delta x=1^{\circ}$ by default to map out the stress fields, requiring a minimum of three events per bin. This low cut-off in terms of numbers will make stress inferences somewhat noisy, but we found that the patterns detected are consistent and mainly smoothly varying from neighboring bins when compared with larger event number binning. For an estimate of the "stationary" pre-M9 stress, we consider all catalog events up to some end time, $t_{e}=2007$ by default.

Given the spatio-temporally clustered nature of seismicity, each spatial bin's inversion or summation result might be dominated by large earthquake aftershock series, for example. For the long-term stress estimates, we therefore weigh each earthquake within a sliding, two-month window by the inverse of the total number of events in that window before inverting to achieve a better approximation of the stationary background stress. For time-variable stress, we use a sliding window weighing all events equally within each bin before the nominal time of consideration for better temporal resolution, with temporal bin width of $\Delta t=3 \mathrm{yrs}$ by default. Any time-dependent quantity analyzed here refers to the end time of the $\Delta t$ interval. We explore variations in $\Delta t$ and $\Delta x$ below.

\subsubsection{Analysis of stress and strain tensors}

There are a range of ways of examining stress tensors, $\sigma$, but we find it helpful to plot moment tensor symbols and indicate their stress types with color by their mean, horizontal stress component

$$
\sigma_{m}=\frac{\sigma_{E E}+\sigma_{N N}}{2}
$$


where $E$ and $N$ are East and North directions, respectively. Negative and positive values indicate horizontal compression and tension, respectively, with shear in between. We also show major, compressive axes for the horizontal stress components, $\vec{\sigma}_{3}$, and the square root of the second tensor invariant,

$$
\tau_{I I}=\sqrt{\frac{\sigma_{E E}^{2}+\sigma_{N N}^{2}+\sigma_{U U}^{2}+2\left(\sigma_{E N}^{2}+\sigma_{E U}^{2}+\sigma_{N U}^{2}\right)}{2}}
$$

as an indication of shear stress, with $U$ for the up direction.

Seismicity derived tensors here do not contain magnitude information and are normalized such that

$$
|\boldsymbol{\sigma}|=1
$$

with tensor norm

$$
|\boldsymbol{\sigma}|=\sqrt{\sum_{i} \sum_{j} \sigma_{i j}^{2}},
$$

and $i, j=\{E, N, U\}$. If we denote normalized tensors as

$$
\hat{\sigma}=\frac{\sigma}{|\sigma|}
$$

then typically $\boldsymbol{\sigma}=\hat{\boldsymbol{\sigma}}$, and for normalized stress tensors, $\sigma_{m} \in[-0.5 ; 0.5]$. (Moment tensor, $\boldsymbol{M}$, components relate to scalar moment $M_{0}=\frac{1}{\sqrt{2}}|\boldsymbol{M}|$.)

We quantify the difference of inferred stress at each time, $\boldsymbol{\sigma}(t)$, compared to the long term stress, $\sigma^{b g}$, in two ways. We compute the stress anomaly,

$$
\boldsymbol{\Delta} \boldsymbol{\sigma}(t)=\boldsymbol{\sigma}(t)-\boldsymbol{\sigma}^{b g},
$$

or, based on normalized tensors for our analysis,

$$
\widehat{\boldsymbol{\Delta} \boldsymbol{\sigma}}(t)=\hat{\boldsymbol{\sigma}}(t)-\hat{\boldsymbol{\sigma}}^{b g}
$$

We then plot $\widehat{\Delta \sigma}$ as log-scale moment tensors, to illustrate all stress anomaly tensor components, and we also show $\Delta \sigma_{m}$ based on the $E E$ and $N N$ components of $\widehat{\Delta \sigma}$ in analogy to eq. (1).

We also show an interpolated field representation where the similarity between current stress and long-term is further reduced to a single, orien- 
tational difference metric, the normalized tensor dot product

$$
\theta=\frac{\boldsymbol{\sigma} \cdot \boldsymbol{\sigma}^{b g}}{\left|\boldsymbol{\sigma} \| \boldsymbol{\sigma}^{b g}\right|}=\frac{\sum_{i, j} \sigma_{i j} \sigma_{i j}^{b g}}{\left|\boldsymbol{\sigma} \| \boldsymbol{\sigma}^{b g}\right|}=\hat{\boldsymbol{\sigma}}(t) \cdot \hat{\boldsymbol{\sigma}}^{b g} .
$$

Values of similarity of $\theta=1$ and -1 then correspond to perfect alignment and complete stress-state reversal, respectively. We also evaluate the spatial mean of $\theta,\langle\theta\rangle$, and the standard deviation from the mean. Stress in eqs. (1)(5) is replaced by the strain or strain-rate tensor, $\varepsilon$, for Kostrov summations.

\subsection{Mechanical model}

To compare the seismicity-based stress estimates with predictions from a physical model, we use the afterslip and visco-elastic relaxation contributions (from co- to post-seismic) estimated from two visco-elastic, 3-D finite element (FE) models. Hashima et al. (2016) explored co-seismic slip inversions for Tohoku-oki and computed elastic Green's functions in the presence of heterogeneity. The resulting slip distribution and the Green's functions for the effect of slip on the plate boundary interface were also used to infer the afterslip component for Freed et al. (2017). Freed et al. fit the cumulative post-seismic geodetic displacements three years after the M9 event using a superposition of static afterslip and a time-dependent component due to visco-elastic relaxation.

The two FE models are near-identical in their structure, but we noticed that the elastic shear moduli used in these models require the visco-elastic contribution's moduli to be scaled up by a factor of 1.5 for stress consistency, partly due to small geometric differences. This correction does not affect any homogeneous medium slip inversions (since geodetic displacements, rather than stress-based quantities were used as data), and even in the presence of strong heterogeneity, inversion results for slip should be affected by $\lesssim 10 \%$ (Hashima et al., 2016). The visco-elastic model of Freed et al. (2017) was thus recomputed with both shear modulus and viscosities scaled up by 1.5 to have the same Maxwell decay time as in the original model.

Besides the rescaling of the visco-elastic contribution, we also modify the details of the afterslip contribution from that used in Freed et al. (2017) since it had no explicit time-dependence. Moreover, we noticed an artifact due to poorly constrained and likely unrealistic afterslip on the Izu-Bonin trench segment. Our new inversion for afterslip suppresses this slip contribution but leads to near identical geodetic displacements. We use this cleaned up version of the afterslip stress contribution. 
For the time-dependence, afterslip-related stresses are here assumed to approach their full amplitude from Freed et al. by means of an asymptotic relaxation function

$$
\alpha(t)=1-\exp \left(-t / t_{p}\right)
$$

where the relaxation time, $t_{p}$, is chosen as 3 yrs, for simplicity. The $t_{p}$ value had not been constrained geodetically and is, in a first step, only meant to explore some time-dependence during the time-period of consideration. Other choices for $t_{p}$ and an alternative, logarithmic form for $\alpha(t)$ are explored in Supplementary Figure 1. These choices play a minor role since afterslip only affects a limited region of the overall crustal stress field, as shown below.

We will refer to this modified mechanical model set as the modified model based on Freed et al. (2017) subsequently, noting that it is fully consistent with the geodetic inversion and conclusions of the original work.

\section{Results and discussion}

Figure 1 reviews some general seismo-tectonic indicators for the wider study region for context. Subduction of the Pacific and Philippine Sea plate occurs underneath NE Japan encompassing northern Honshu and Hokkaido and the Japan and Kuril trenches. Based on large events in the gCMT catalog (Ekström et al., 2012) (Figure 1) this setting displays the classic pattern of outer rise extension and transition into thrust faulting, as one moves from the oceanic plate across the trench to the overriding plate. This deformation pattern transitions to right-lateral shear deformation on land toward the SW along Shikoku and Kyushu (e.g. Seno, 1999; Terekawa and Matsu'ura, 2010; Loveless and Meade, 2010). While the central part of the Nankai trough has seen large earthquakes historically, it appears relatively devoid of thrust events over the past few decades (Figure 1).

\subsection{Long-term, reference stress-state of the crust pre M9}

Figure 3 shows a zoom-in of more detailed, long-term crustal stress inference based on a $\Delta x=1^{\circ}$ binning of normalized moment tensors from all F-net events with $M>3$ up to 2007 (Figure 2) for a Kostrov summation (a) and when using the same events to infer stress using a Michael (1984) inversion (b), each weighted by the inverse of event number within two month bins over time. As seen for the large events of Figure 1, most of NE Japan is inferred to have been under horizontal compression before the M9. Relative, long-term rigid plate convergence provides a good first guess for 

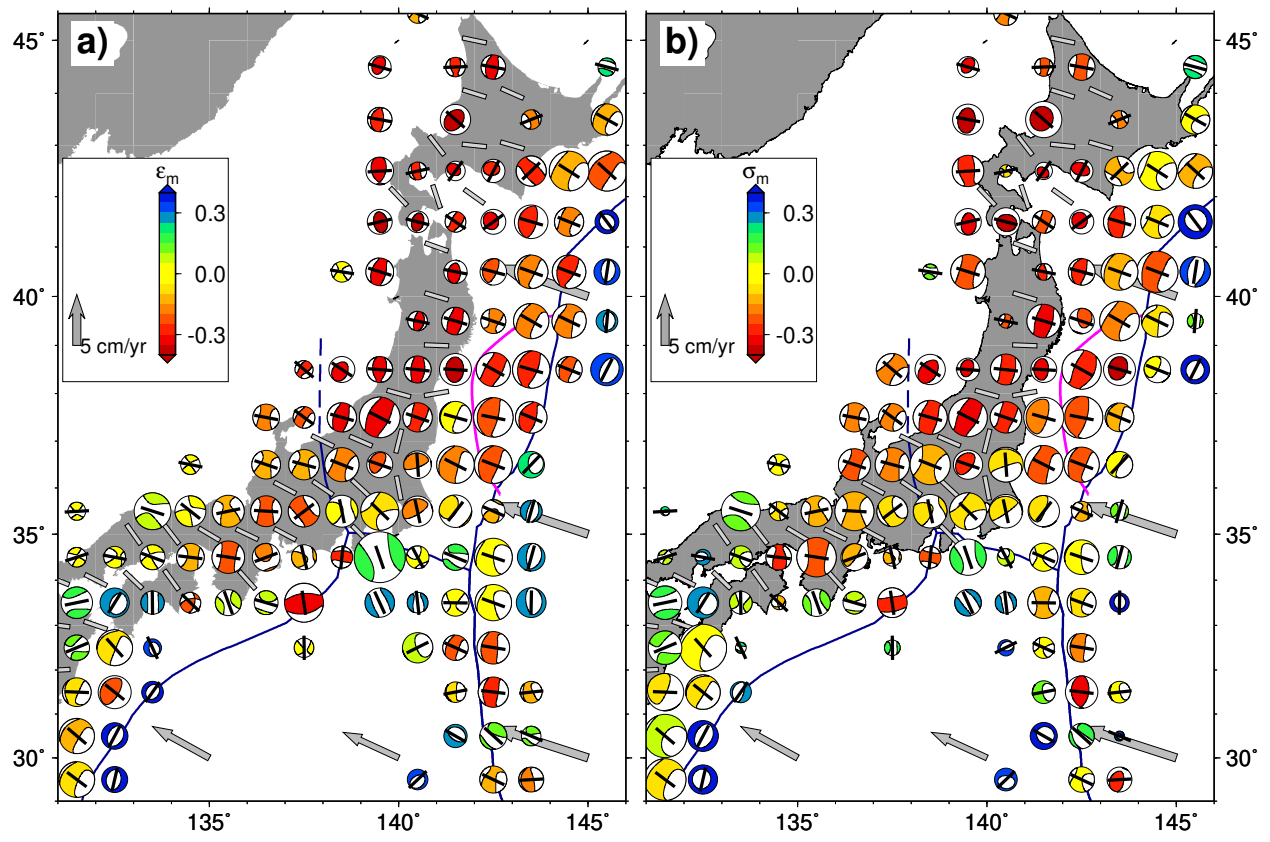

Figure 3: Long-term, pre-M9 crustal stress from F-net moment tensors (Okada et al., 2004) (depths $z \leq 36 \mathrm{~km}$, Figure 2), binned on a $\Delta x=1^{\circ}$ grid, up to 2007. We use all spatial bins with at least three events and weigh each event inversely to the number of events per two month temporal bin. a) shows a normalized Kostrov (1974) summation, b) a Michael (1984) stress tensor inversion. Moment tensors are colored with the mean horizontal strain/stress, $\varepsilon_{m}$ and $\sigma_{m}$, respectively. Symbol sizes scale with $1+\log (N)$ with $N \geq 1$ the event number per bin (a) and inversely with the square root of the norm of the uncertainties of the stress tensor inferred from a Monte-Carlo estimate swapping likely fault planes (b, cf. Michael, 1987). Black sticks denote the orientation of the major compressive strain/stress axis, $\vec{\sigma}_{3}$, of the horizontal components. Gray arrows are MORVEL (Argus et al., 2011) plate motions with respect to the Amur plate. Gray sticks on land are major compressive strain-rate orientations inferred from computing gradients of $1^{\circ}$ averaged geodetic velocities. Velocities were computed by linear fits of GEONET GPS daily solutions up to 2007. Magenta contour outlines the $\geq 5 \mathrm{~m}$ co-seismic slip area for Tokoku-oki M9 from Hashima et al. (2016) for reference. 
the orientation of the major compressive axis of the horizontal components that is inferred here (e.g. Terekawa and Matsu'ura, 2010).

Convergent zones such as large parts of Japan are clearly not rigid plates on the scales of Figure 1, however, but show significant crustal deformation over hundreds of kilometers away from the trench. On land, we can thus further compare the crustal stress state with geodetically inferred strainrates, whose style is shown in Figure 3 based on Geospatial Information Authority (GSI) GEONET (Sagiya, 2004) time-series up to 2007. Orientations of inferred $\vec{\sigma}_{3}$ on land are overall comparable to the geodetic strainrate $\overrightarrow{\dot{\varepsilon}}_{3}$ (e.g. Sagiya, 2004; Townend and Zoback, 2006; Terekawa and Matsu'ura, 2010; Loveless and Meade, 2010), with some regional exceptions such as on Hokkaido and Kyushu (Savage et al., 2016).

An overall match between stress from seismicity and strain-rates from geodesy is often found in tectonically active regions with distributed deformation. However, it is expected to be perturbed temporally by both regional earthquakes (e.g. Becker et al., 2005) and the megathrust earthquake cycle in subduction zones (e.g. Wang, 2000), including aforementioned variations in the loading at the plate boundary (e.g. Loveless and Meade, 2016), for example.

From comparing the normalized Kostrov summations and stress inversions (Figures $3 a$ and $b$ ), we can see that the orientations of the major horizontal compressive axes of strain and stress tensors are overall aligned very well, as expected. This implies that the effects of mechanical anisotropy are generally minor on the averaging scales of analysis. The mean horizontal normal components (moment tensor coloring in Figure 3) are more variable between the analysis methods, however. This likely reflects the different assumptions inherent in the binning of normalized moment tensors vs. resolving stress on a set of heterogeneous fault planes from a double couple approximation. In limited regions, the stress field as visualized by the moment tensor symbols in Figure $3 b$ indicates a large non-double couple component (e.g. uniaxial compression or (ex)tension "doughnut" girdles close to the Japan trench at $\sim 38.5^{\circ} \mathrm{N}$ ). This does, of course, not mean that deformation is locally necessarily accommodated in this style, but rather that a superposition of different shear type of faulting overall amounts to such a stress state when averaged over large spatial scales (cf. Bailey et al., 2010).

Considering the distribution of event numbers, $N$ (Figure 3a) or uncertainties inferred from randomly choosing the active fault plane from a double-couple pair (Figure 3b), patterns for these two measures of robustness for strain/stress estimates are overall consistent with stress un- 

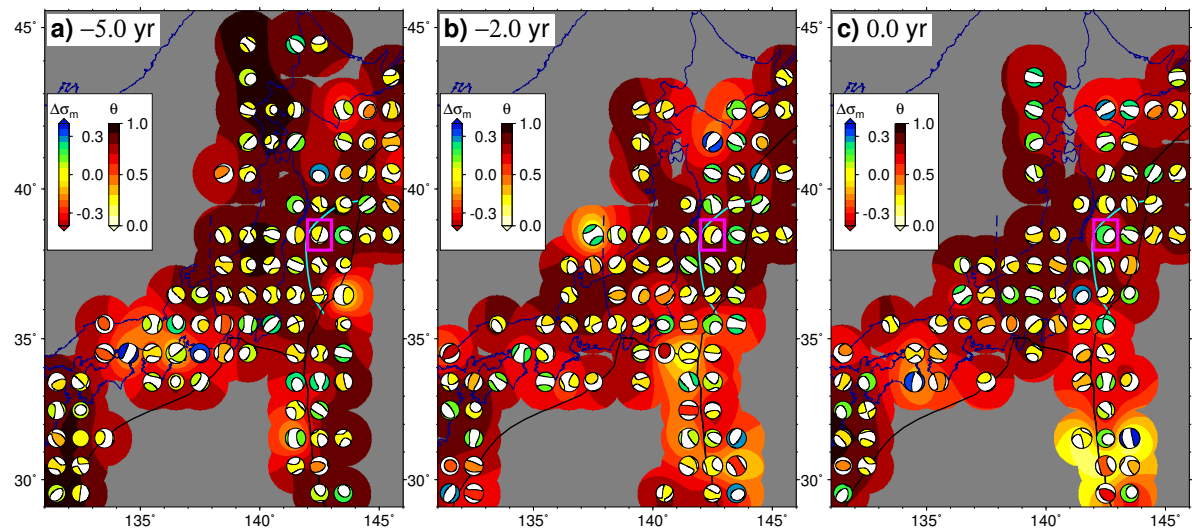

Figure 4: Time evolution of crustal background stress anomaly before the M9 Tohoku-oki earthquake on March 11, 2011. Inference is based on a $\Delta t=3 \mathrm{yr}$, sliding time-window Michael (1984) stress inversion on a $\Delta x=1^{\circ}$ grid. Moment tensor symbols indicate the stress tensor difference, $\widehat{\Delta \sigma}$ (eq. 4), from long-term (Figure 3b), with the horizontal normal stress anomaly component, $\Delta \sigma_{m}$, colored, using a log-scaling for the size of moment tensors. Labels indicate the end-time of each window relative to the occurrence of the M9, with $t=0.0 \mathrm{yr}$ (c) just before the earthquake. Background shows the normalized tensor dot product, $\theta$ of eq. (5), for the similarity between time-local stress inference and the long-term stress (unity indicating perfect alignment). Cyan contour is the inferred $\geq 5 \mathrm{~m}$ co-seismic slip region of the M9 from Hashima et al. (2016) for reference, and magenta box denotes the site selected for analysis in Figure 6. See Figures 7a-c for temporal perturbation due to and after the M9.

certainty scaling roughly with the inverse of $\log ^{2}(N)$. There are some differences in estimates of robustness in regions where large event numbers mask redundancy of information about the stress state. Coverage is poor in northernmost Honshu and Hokkaido, adequate in central Honshu, and best in regions of clustered seismicity such as around the future M9 fault area.

\subsection{Time-dependent crustal stress}

Figure 4 shows three snapshots of the inferred differences between the time-dependent stress state and the long-term, pre-M9 state of Figure 3, evaluated at 5 and 2 years before the 2011 M9, as well as just before, respectively. The maps of Figure 4 are based on using a sliding, $\Delta t=3 \mathrm{yr}$ 
window of catalog seismicity for the stress binning, implying much lower numbers of events in each bin compared to the long-term estimate. Earthquakes whose deformation patterns do not match the long-term estimate of Figure 3 perturb the regional stress field slightly at all times, reflecting the spatio-temporally clustered nature of seismicity. This leads to large variability and complicates establishing meaningful trends somewhat. The standard deviation of tensor similarity, $\theta$ of eq. (5), is $\sim 0.2$ for the maps shown in Figure 4 (cf. Figure 5). However, the fluctuations of the stressstate in terms of the mean, horizontal stress anomaly component is modest, with $\Delta \sigma_{m} \lesssim 0.15$ typically.

Besides these fluctuations in pre-M9 stress whose tectonic or volcanic significance is unclear, there also appears to be a subtle trend of decreasing similarity to the long-term stress state around the future Tohoku-oki fault plane, indicated by a region where $\theta$ is decreased to $\sim 0.75$ throughout much of NE Japan (Figure 4c), relative to stress inferred up to 2007. Here, we define NE Japan as the Amur plate region East of the Itoigawa-Shizuoka Tectonic Line and its offshore extension (Figure 2) and Northern Honshu as the on-land subset of that region South of $41^{\circ} \mathrm{N}$.

To explore this subtle change in stress state further in a more statistical way, Figure 5 shows the spatially averaged tensor dot product, $\langle\theta\rangle$, of sliding time window estimates of stress compared to long-term, as in Figure 4, for \pm 7 yrs around the M9 based on stress inversions and moment tensor summation at $\Delta x=1^{\circ}$ and $\Delta t=3 \mathrm{yr}$. The progressive deviation of the match to long-term stress before the M9 close the eventual fault plane is seen in a near-monotonous decrease of $\langle\theta\rangle$ for NE Japan by $\sim 0.1$ (Figures $5 b$ and e).

Clear trends of type of change in stress state (e.g. horizontal tension to compression) before the M9 are hard to reliably detect in much of the study region. However, a bin on top of the future M9 fault slip area shows a subtle, but systematic increase in $\Delta \sigma_{m}$, i.e. a trend toward larger degrees of tension (magenta box in Figure 4). Figure 6 tracks the total, normalized stress state for this location over time, and explores a range of $\Delta t$ values for which the deviation from long-term compression is seen to consistently commence around 2007. A similar change of stress before the M9 is seen to the SW of this particular bin (Figure 4).

Such a pre-M9 stress field modification might relate to the change in geodetically inferred deformation state of the overriding plate close to the M9 as analyzed by Ozawa et al. (2012) and Mavrommatis et al. (2014), and recently explored in terms of temporal evolution of coupling by Iinuma (2018). Another contribution to the stress field change as seen in Figure 6 

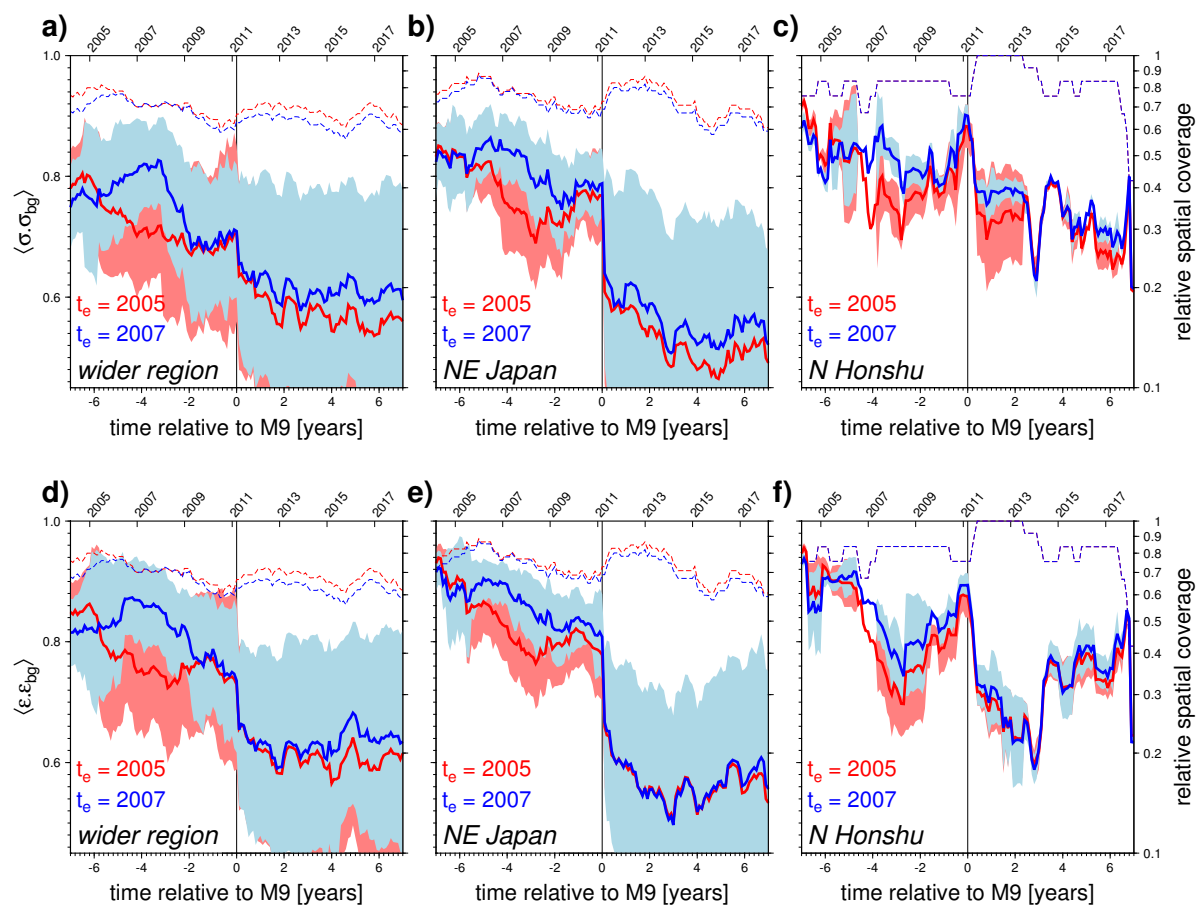

Figure 5: Spatially-averaged, normalized tensor dot product $(\langle\theta\rangle$, eq. (5), solid lines) and fractional area fill (dashed lines) compared to long-term for a $\Delta x=1^{\circ}, \Delta t=3$ yr sliding window for the whole region as in Figure 4 (a and d), when limited to the NE Japan region (b and e, defined as $\mathrm{N}$ of the ISTL and its offshore extension, Figure 2), and when further spatially limited to regions on land and south of $41^{\circ} \mathrm{N}$ (c and $\mathrm{f}$, "Northern Honshu", cf. Figure 1). End of long-term summation values, $t_{e}$, of 2005 and 2007 are shown. Shaded background range indicates \pm 0.5 the spatial standard deviation of $\theta$ from $\langle\theta\rangle$. Plots a)c) are for Michael (1984)-type stress inversions, and d)-f) for normalized Kostrov (1974) summations. See Supplementary Figure 2 for other choices of spatial and temporal binning. 


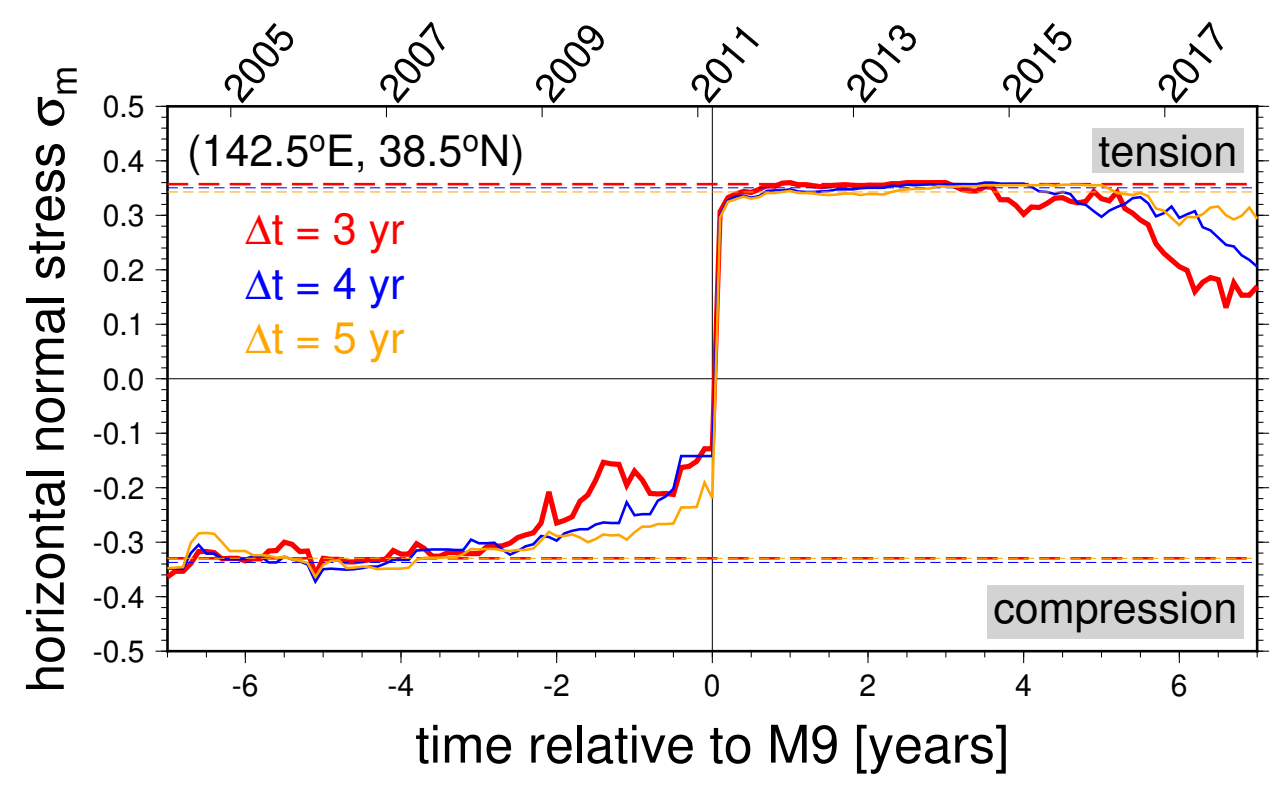

Figure 6: Mean horizontal stress, $\sigma_{m}$, vs. time, $t$, for an example location on top of the M9 fault slip area (see magenta box in Figures 4 and 7) from Michael (1984) type inversion, for $\Delta t=[3,5]$ yrs (stress anomaly shown in Figures 4 and 7a-c is for $\Delta t=3 \mathrm{yr}$ ). Note deviation from presumed long-term, pre M9 compressive state (lower dashed lines show mean $\sigma_{m}$ for $t<-4 \mathrm{yr}$, cf. Figure $3 \mathrm{~b}$ ) starting at $\sim 2007$ (cf. Figure 5b), large jump due to the co-seismic effect of the M9 (cf. Yoshida et al., 2012; Hasegawa et al., 2012), indication of a short term-transient further increase of $\sigma_{m}$ over $\sim 1 \mathrm{yr}$ until a plateau is reached at $t \sim 1 \mathrm{yr}$ (upper dashed lines show mean $\sigma_{m}$ for $1 \leq t \leq 3 \mathrm{yr}$ ), and ongoing reduction of tension and possible eventual recovery of the compressive long-term state starting at $\sim 2015$. 
might be a series of large earthquakes between 2003 and 2011 whose contributions to geodetically detected deformation was analyzed by Suito et al. (2011) and Johnson et al. (2016). It remains to be determined if the associated stress change of those events would be large enough to explain the post 2007 stress field modification, and if these events should be considered independent of any change in plate boundary coupling.

The biggest signal in the time-dependent stress change of Figures 5-7 is, as expected, due to the M9 itself. The Tohoku-oki earthquake is seen to have changed the stress field from the long-term abruptly by a drop of $\langle\theta\rangle$ of $\Delta\langle\theta\rangle \sim 0.1 \ldots 0.2$. This modification of the crustal stress was not just limited to regions close to the rupture, but is also seen regionally onshore in Honshu, for example (Figures $5 \mathrm{c}$ and $\mathrm{f}, 7 \mathrm{a}$ and $\mathrm{b}$ ), substantiating the analysis of Yoshida et al. (2012) and Hasegawa et al. (2012). Temporal trends between stress inversions and Kostrov summations are generally consistent (Figure 5), but somewhat less spiky and apparently more clearly related to the M9 in the Kostrov summations. The standard deviation fluctuations for similarity, $\theta$, seen in map view for Figure 4 are represented by large fluctuations around the mean, $\langle\theta\rangle$, (shading in Figure 5). Those are reduced when shrinking the region of averaging from the whole study domain, to the NE Japan region, and further to northern Honshu (Figures 5a through c). This indicates that $\langle\theta\rangle$ is a more meaningful metric on those smaller scales.

One of the complications of such a time-dependent stress field analysis is that any crustal earthquakes that are not reflective of the long-term stress as defined in Figure 3 and their aftershocks will offset the stress field in ways that are possibly unrelated to the M9. Another problem arises because the coverage of the time-variable stress maps is variable to some extent, as shown in the dashed lines in Figure 5, compared to the long-term area fill of Figure 3. There is some correlation of trends in $\langle\theta\rangle$ with the fractional area coverage, particularly when considering the whole study area (Figures $5 \mathrm{a}$ and $\mathrm{d}$ ). However, the sign of this correlation is not always the same (i.e. a decrease in $\langle\theta\rangle$ can be accommodated by both an increase or decrease of spatial coverage), and the more regionally focused analysis (Figures $5 \mathrm{c}$ and $\mathrm{f}$ ) of $\langle\theta\rangle$ appears mostly independent of time-variable spatial coverage.

Keeping such complexities in mind, we can attempt to interpret the inferred changes in stress-state beyond the co-seismic step-modification. Considering NE Japan, we can see a long-term drop of the stress and strain similarity compared to long-term starting around 2007 and continuing to the M9 event (Figures 5b and e), as was discussed for Figure 4 and perhaps best illustrated by Figure 6. When considering northern Honshu, the drop 

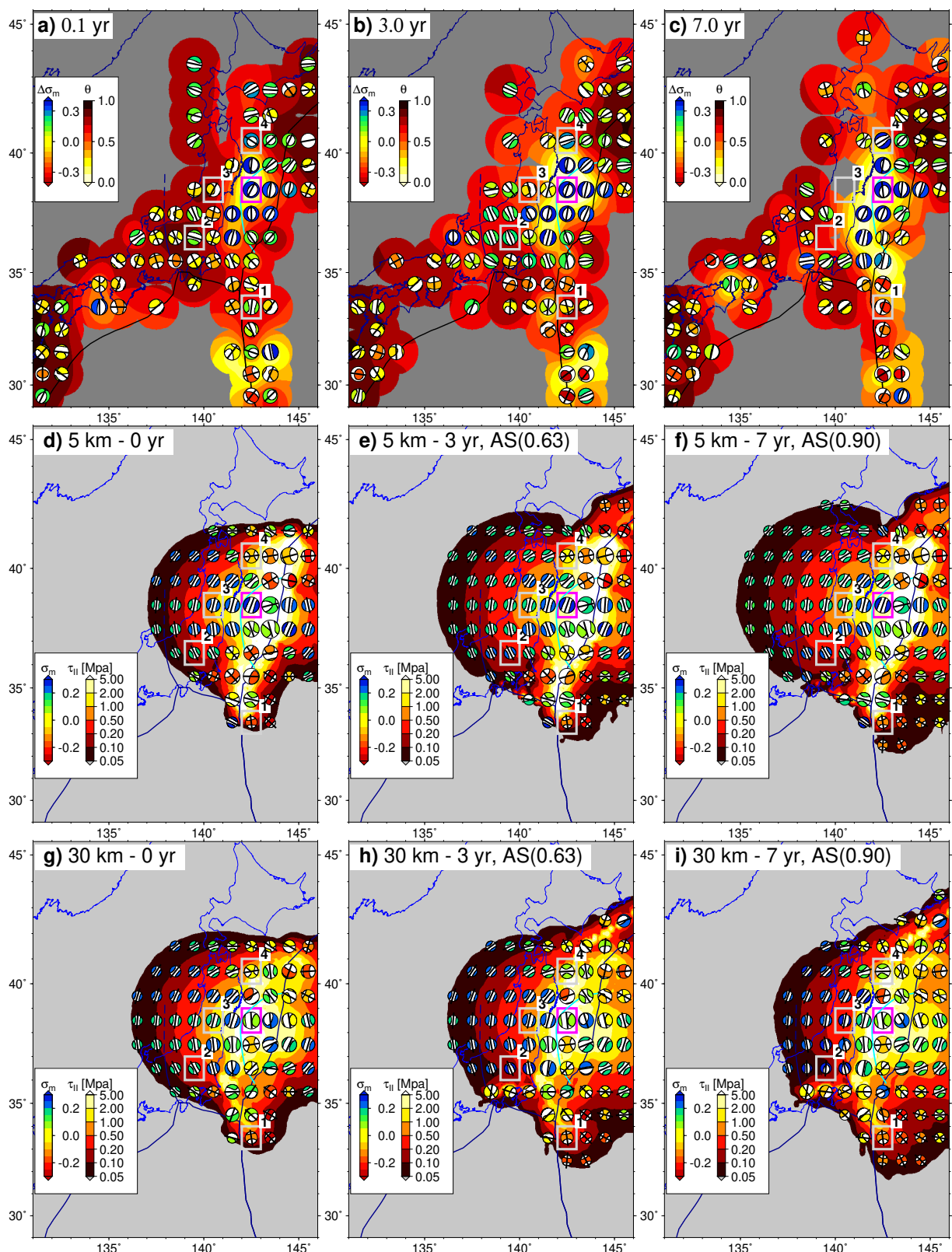

Figure 7: a) - c): Crustal stress anomaly, $\widehat{\Delta \sigma}$, evolution after the $2011 \mathrm{M} 9$, continued from Figure 4. The local example of Figure 6 is shown as a magenta box. Gray numbered boxes indicate other bins discussed in the text. d) - i): Time evolution of the visco-elastic model stress at $5 \mathrm{~km}$ (d-f) and $30 \mathrm{~km}$ (g-i) depths of the modified Freed et al. (2017) model. Background shows the shear stress, $\tau_{I I}$ of eq. (2), of the model stress tensor, and moment tensors are only shown for regions with $\tau_{I I} \geq 0.05 \mathrm{MPa}$. The afterslip contribution ("AS" fraction of full afterslip stated in legend) is computed from eq. (6) with $t_{p}=3$ yr. Sticks indicate the orientation of the major compressive axis, $\vec{\sigma}_{3}$, of horizontal stress. 
and potential intermediate recovery of $\theta$ is less clear. The details of any such trends will depend on choices of $\Delta t$ and $\Delta x$ whose resulting spatiotemporal volume govern the trade-off between robust, potentially oversmoothed, and noisy, possibly under-constrained estimates. Any binning or smoothing of stress inferences based on seismicity may also lead to a sampling bias if the stress state is heterogeneous (e.g. Yang et al., 2013) and different fault systems are activated by the M9 and its aftershock that were not reflected in the pre-M9 stress. Supplementary Figure 2 shows the range of $\Delta t=1 \ldots 7 \mathrm{yr}$ and $\Delta x=0.5 \ldots 2$. We selected $\Delta x=1^{\circ}$ to avoid over smoothing spatially (cf. Figure 4 ), and $\Delta t=3$ yr because further extension of the temporal bins led to smoother, but generally consistent, trends compared to Figure 5.

Besides choices on spatio-temporal binning, any comparison of timedependent stress with some "stable" reference will of course depend on the definition of the stable time period, as is the case for GPS time-series. Comparing $t_{e}$ cases for 2005 and 2007 for the whole study region (Figure 5a and $\mathrm{d}$ ), the end point of summation does indeed control the start time of deviation of $\langle\theta\rangle$ from $\sim 0.8$ to $\sim 0.6$ before the M9. However, focusing on NE Japan and northern Honshu (Figure $5 b, c, e, f$ ), the $\langle\theta\rangle$ trends are consistent for different choices of summation end times (the $t_{e}=2009$ case behaves similarly, cf. Supplementary Figure 2). This implies that the finding of stress-state modification on a system wide level due to M9 is robust. Close to the fault zone, geodetically determined decadal transients (e.g. Ozawa et al., 2012; Mavrommatis et al., 2014) therefore appear accommodated by a likely related crustal stress-state transient before the M9.

Considering the time-dependence of inferred crustal stress after the M9, we can see a sustained offset from the long-term stress (decrease in $\langle\theta\rangle$ ), particularly for NE Japan (Figures $5 b$ and $d$ ), with a possible indication of a reversal and recovery of the pre-M9 stress state around 2015 (particularly clear in Figure 5e and f). This might indicate loading of the crust in a style consistent with co-seismic slip due to afterslip on short $(\sim$ one year) timescales, and then perhaps dominance of visco-elastic relaxation on longer timescales. This observation motivates our comparison of the inferred stress state with model predictions from the modified mechanical model of Freed et al. (2017), and is shown in map view in Figures 7a-c (in continuation of Figure 4).

Comparing the stress anomalies, it is clear that $\widehat{\Delta \sigma}$ amplitudes are much larger, and presumably significant, for the co- and post-seismic sequence than the lead up to the M9 (see, e.g., $\Delta \sigma_{m}$ values). The region between 
northern Honshu and the Japan trench that were previously strongly compressive in the horizontal stress component (Figure 3) are much more in tension after the M9, and there is an ellipsoidal region around the M9 fault plane within northern Honshu that indicates tension mixed with stress that would lead to shear. The stress modification due to the M9 is, as expected from our understanding of the megathrust cycle within a geodetic context (e.g. Wang, 2000; Wang et al., 2012), large enough to not only produce tensional normal stress change, $\Delta \sigma_{m}$, in the horizontal as in Figure 7a-c, but the total mean horizontal stress, $\sigma_{m}$, jumps into tension as well (Figure 6).

Besides the flip in the sign of $\sigma_{m}$, this M9-proximal location analyzed in Figure 6 also nicely illustrates that the stress-state as inferred from seismicity changed before the M9 starting at $\sim 2007$, and that there appears to be an indication of possibly visco-elastic recovery of the long-term state commencing at $\sim 2014$, as was discussed for Figure 5 . While perhaps too subtle a feature to conclusively interpret, Figure 6 also shows a $\sim 1$ yr transient just after the M9, possibly related to afterslip. This behavior is overall robust with respect to the choices of $\Delta t$ for the range that enhances temporal smoothness ( $\Delta t \geq 3 \mathrm{yrs}$ as in Figure 6), besides some dependence on $\Delta t$ because of edge effects of the M9 and time-series limitations.

Considering the spatial patterns of $\theta$, the low $(\theta \sim 0)$ anomalies that indicate significant stress tensor reorientation within the crust start close to the M9 fault plane for the co-seismic effect (Figure 7a), and then spread onto northern Honshu NW five years after the M9 (Figure 7b). At the end of our study period, the region with $\theta \sim 0$ is somewhat narrower (Figure 7c), indicating slightly reduced $\theta$ anomaly along the outer rise, and a shift of reduction in $\theta$ toward the south along the Izu-Bonin trench. There, the region south of $31^{\circ} \mathrm{N}$ is affected from a presumably unrelated earthquake before the M9 (Figure 4c), making it difficult to distinguish cause and effect.

\subsection{Modeled stress change due to the M9 event}

The significant changes in crustal stress as imaged by seismicity due to both co- and post-seismic effects motivate us to compare the observations discussed in the previous section to modeling results. Given the aforementioned problems with potential sampling bias of seismicity and complexities in the interpretation of stress inversions, we do not expect that all of the apparent stress state modification is due to mechanical loading changes from the M9. Nonetheless, it is instructive to explore which aspects of the inferences may be linked to deterministic modeling in lieu of more detailed information on fault structures and the possible rheological heterogeneity in the crust. 
Figures 7d-i show time-dependent stress from the visco-elastic plus afterslip approach of the modified Freed et al. (2017) model, evaluated for the first seven years after the M9. These stresses would be perturbations to the background stress and are expected to lead to a differential effect, comparable to our stress anomaly inferences of Figures 7a-c if the crustal background stress is of comparable amplitude, which we discuss further below.

Tracking the expanding front of significant, M9 related shear stress, $\tau_{I I}$ of eq. (2), at shallow depths (5 $\mathrm{km}$ in Figures $7 \mathrm{~d}-\mathrm{f})$, we can see how the deeper viscous relaxation within the mantle leads to an elastic loading of the shallow crust with perturbations of order $\sim 1 \mathrm{MPa}$ within Honshu. The initially mainly tensional stress perturbation $\left(\sigma_{m} \gtrsim 0.2\right)$ in the $\mathrm{W}$ and $\mathrm{E}$ of the M9 rupture shows reduced $\sigma_{m}$ over time, and visco-elastic reloading turns the modeled stress state into more of a strike-slip character (e.g. box 3 in Figures 7d-f).

There is also a spatial widening of the modeled shear stress, $\tau_{I I}$, perturbation toward the south along the Izu-Bonin trench due to viscous stress redistribution (e.g. box 1 and south of it). The afterslip contribution leads mainly to perturbations offshore and close to the M9 fault plane (Figure 8). Supplementary Figure 1 shows that different choices for the afterslip timescale $t_{p}$ or a logarithmic form for the afterslip time-dependence, $\alpha(t)$, lead to very similar results, with the most pronounced difference perhaps in the inferred $\sigma_{m}$ state toward the south of the M9 rupture.

Neither the visco-elastic nor afterslip time-dependence are meant to directly match the stress field inferences for our study. We are mainly concerned with the overall process and defer a more detailed attempt to match the stress field evolution to later visco-elastic modeling work which captures the GPS geodetic time-series fully, rather than considering cumulative post-seismic displacements as was done by Freed et al. (2017).

Considering the modeled stress perturbations at larger depths $(30 \mathrm{~km}$ in Figures 7g-i) we see the opposite behavior compared to $5 \mathrm{~km}$ depth, as expected from visco-elastic modeling of megathrust post-seismic deformation: the co-seismic stress is relaxed westward of the M9 rupture (within the hanging wall), and the stress perturbation shifts seaward behind the fault over time. Comparing the stress state, the deeper layers are predicted to have less of a strike-slip type component than the shallow crust but the general patterns, e.g. of major compressive axes, are similar except close to the M9 fault plane.

When comparing the stress anomaly from seismicity with our model results, the depth distribution of the F-net catalog (Figure 2) leads us to 
expect that the $36 \mathrm{~km}$ layer average of Figures 7a-c to be dominated by shallower seismicity on land (e.g. boxes 2-4 in Figures 7d-f) and deeper events offshore (e.g. box 1 and east of the slip area in Figures 7g-i). With this possible bias in mind, we find that both observations and model show the major signal of relative tension due to the $\mathrm{M} 9$ co-seismically close to the fault plane, and subsequent reduction in tensional stress (Figure 6).

Additional similarities exist in the major compressive axis, $\vec{\sigma}_{3}$, orientations (e.g. boxes 2-4). The region that is inferred to have been put under relative tension appears somewhat more N-S oriented in the observations compared to the model. South of the rupture zone, the shift of stress field modification toward the Izu-Bonin trench (Figure 7c) is likewise found in both stress inversions and model results, with similar $\vec{\sigma}_{3}$ orientations (e.g. box 1). On land in northern Honshu, there is also a broad match between inferred and predicted stress change $\vec{\sigma}_{3}$ such as within box 2 of Figure 7, and box 4 of Figures $7 b$ and c, where the ellipsoidal trajectories of the model stress perturbation appear reflected in a change of the style of seismicity.

We therefore suggest that both co- and post-seismic stress change as predicted by the modified model of Freed et al. (2017) (and, by inference, any similar model that is able to match the geodetic constraints) provides a good first-order description of the change in crustal stress seen immediately due to and after the Tohoku-oki earthquake. This implies that joint geodetic and stress inversions for deformation models may be meaningful even in megathrust settings (cf. Becker et al., 2005). Of course, this is only true if the perturbations due to the model actually modify crustal stress or stressing-rates significantly. If we assume that Michael (1984) type focal mechanism inversions do indeed image stress, rather than stressing-rate as has sometimes been suggested (Twiss and Unruh, 1998; Smith and Heaton, 2011), this means that the background stress levels are comparable to the far-field perturbations, which are only fractions of a $\mathrm{MPa}$ across parts of Honshu (e.g. Figure 7f).

Figure 8 shows how the long-term stress would be affected in the whole region and northern Honshu in terms of the mean tensor dot product, $\langle\theta\rangle$, for comparison with the actual variations of Figure 5. The postseismic stress state is computed by adding the long-term stress state tensors, e.g. as in Figure 3, scaled by absolute stress values to Freed et al.'s (2017) modified model stress perturbations, e.g. as in Figure 7d-i, assuming linear superposition is applicable. We then process the stress state in the same way as for the seismicity inferred stresses (e.g. Figures 4 and 5).

As would be inferred from the perturbations alone (Figures $7 d-i$ ), the shallow levels of the crust are predicted to experience a long-term modi- 


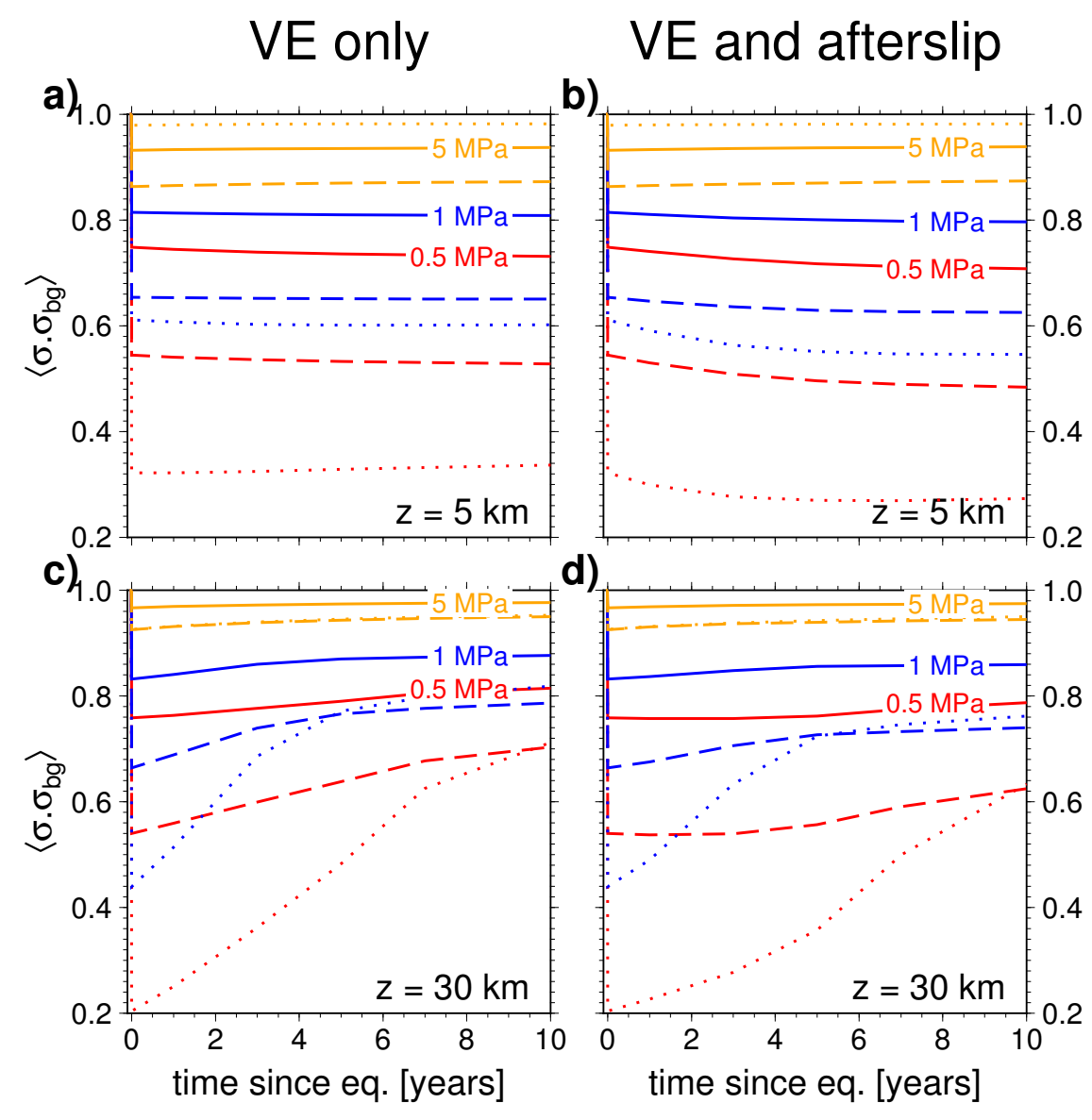

Figure 8: Predicted modification of the inferred stress state in terms of mean tensor dot product, $\langle\theta\rangle$, for the whole region covered by the long-term stress state inference (solid lines, cf. Figure $3 b$ ) and when restricted to the NE Japan region (dashed line) and northern Honshu (dotted line). Assumed background stress levels of the long-term field are chosen as indicated, and results are shown at shallow (a) and b), as in Figure 7d-f) and larger depth (c) and d), as in Figure 7d-f) for the visco-elastic component of Freed et al.'s (2017) modified model ( $a$ and $c$ ), and when adding the afterslip contribution ( $b$ and $d$ ). 
fication of stress with transients in Figure $8 \mathrm{~b}$ mainly due to the assumed afterslip accumulation. Deeper levels of the crust and upper mantle are already experiencing significant reduction of the M9 effect (Figure 8c) given the effective Maxwell time of Freed et al.'s (2017) visco-elastic model parameters. Models with afterslip contributions predict only a slightly larger perturbation of the stress field than visco-elastic effects alone, consistent with the analysis of Figure 7 that showed that the afterslip related stress modification is mainly seen in limited regions offshore.

Using the regional, NE Japan region co-seismic drop of stress field similarity to long-term of $\Delta\langle\theta\rangle \sim 0.2$ (Figure $5 \mathrm{~b}$ ) as a guide, we would infer a background stress level between $\sim 1 \ldots 5 \mathrm{MPa}$ from the modeled viscoelastic perturbation. This estimate is in line with inferences from co-seismic stress change studies for the M9 (Yoshida et al., 2012) and elsewhere (e.g. Hardebeck and Hauksson, 2001). Figure 8 also reemphasizes that it is the deeper levels of the crust that experience stress evolution curves that are sensitive to the visco-elastic relaxation, providing a potentially useful target for the focus of future, refined inversions.

\section{Conclusions}

We substantiate that the crustal stress field surrounding the 2011 Tohoku-oki M9 earthquake appears to have changed systematically on a regional scale due to the co-seismic rupture effect. We newly find systematic changes in the stress state of the crust over $\sim 4$ yrs before the earthquake, which might be related to geodetically-detected transient coupling along the plate boundary. Following the M9, afterslip appears to enhance the co-seismic stress change locally in diagnostic ways over $\sim$ one year. Mechanical models of visco-elastic relaxation and afterslip based on prior inversions of geodetic constraints capture several of the patterns of stress perturbations and suggest low background deviatoric stress levels of $\sim 5 \mathrm{MPa}$ or less.

At least locally, there is also some indication that $\sim$ four years after the M9, the stress field change has started a trend that appears related to slow reversal and redistribution of the co-seismic M9 perturbation, likely related to viscous relaxation in the crust and mantle. These findings indicate that the crustal stress state as inferred from moment tensor summation or focal mechanism inversion could be inverted jointly with geodetic constraints for comprehensive deformation models of the megathrust cycle. Such efforts have the potential to advance our understanding of time-dependent seismic hazard. 


\section{Acknowledgments}

We thank the National Research Institute for Earth Science and Disaster Resilience and the Geospatial Information Authority of Japan for making the F-net catalog and GPS time-series, respectively, available, and Andy Michael for sharing his stress inversion subroutines via the USGS website. We also thank David Okaya for assistance with the construction of the original visco-elastic FE model, and Kaj Johnson as well as Kelin Wang and an anonymous reviewer for helpful comments on an earlier version of this manuscript. We acknowledge partial support from the U.S. National Science Foundation through EAR-1722680 (TWB and AMF) and the Japanese Ministry of Education, Culture, Sports, Science and Technology through the Integrated Research Project on Seismic and Tsunami Hazards Around the Sea of Japan (AH and HS). All plots were made with the Generic Mapping Tools (Wessel and Smith, 1998), and all processing scripts used for this study are available upon request. 


\section{References}

Argus, D.F., Gordon, R.G., DeMets, C., 2011. Geologically current motion of 56 plates relative to the no-net-rotation reference frame. Geochem., Geophys., Geosys. 12. doi:10.1029/2011GC003751.

Bailey, I.W., Becker, T.W., Ben-Zion, Y., 2009. Patterns of co-seismic strain computed from southern California focal mechanisms. Geophys. J. Int. 177, 1015-1036.

Bailey, I.W., Ben-Zion, Y., Becker, T.W., Holschneider, M., 2010. Quantifying focal mechanism heterogeneity for fault zones in central and southern California. Geophys. J. Int. 183, 433-450.

Becker, T.W., Hardebeck, J.L., Anderson, G., 2005. Constraints on fault slip rates of the southern California plate boundary from GPS velocity and stress inversions. Geophys. J. Int. 160, 634-650.

Bird, P., 2003. An updated digital model of plate boundaries. Geochem., Geophys., Geosys. 4, 1027. doi:10 .1029/2001GC000252.

Ekström, G., Nettles, M., Dziewonski, A.M., 2012. The global CMT project 2004-2010: Centroid-moment tensors for 13,017 earthquakes. Phys. Earth Planet. Inter. 200, 1-9.

Freed, A., Hashima, A., Becker, T.W., Okaya, D.A., Sato, H., Hatanaka, Y., 2017. Resolving depth-dependent subduction zone viscosity and afterslip from postseismic displacements following the 2011 Tohoku-oki, Japan earthquake. Earth Planet. Sci. Lett. 459, 279-290.

Gudmundsson, O., Sambridge, M., 1998. A regionalized upper mantle (RUM) seismic model. J. Geophys. Res. 103, 7121-7136.

Hardebeck, J.L., 2006. Homogeneity of small-scale earthquake faulting, stress and fault strength. Bull. Seismol. Soc. Am. 96, 1675-1688.

Hardebeck, J.L., 2012. Coseismic and postseismic stress rotations due to great subduction zone earthquakes. Geophys. Res. Lett. 39. doi:10 . 1029/2012GL053438.

Hardebeck, J.L., Hauksson, E., 2001. Crustal stress field in southern California and its implications for fault mechanics. J. Geophys. Res. 106, 21859-21882. 
Hardebeck, J.L., Michael, A.J., 2006. Damped regional-scale stress inversions: Methodology and examples for southern California and the Coalinga aftershock sequence. J. Geophys. Res. 111. doi:10.1029/ 2005 JB0 04144 .

Hardebeck, J.L., Okada, T., 2018. Temporal stress changes caused by earthquakes: A review. J. Geophys. Res. 123, 1350-1365. doi:10.1002/ 2017 JB014617.

Hasegawa, A., Yoshida, K., Asano, Y., Okada, T., Iinuma, T., Ito, Y., 2012. Change in stress field after the 2011 great Tohoku-Oki earthquake. Earth Planet. Sci. Lett. 355, 231-243.

Hasegawa, A., Yoshida, K., Okada, T., 2011. Nearly complete stress drop in the $2011 \mathrm{Mw} 9.0$ off the Pacific coast of Tohoku Earthquake. Earth Planet. Space 63, 703-707.

Hashima, A., Becker, T.W., Freed, A.M., Sato, H., Okaya, D.A., 2016. Coseismic deformation due to the 2011 Tohoku-oki earthquake: influence of 3-D elastic structure around Japan. Earth, Planet., Space 68. doi:10.1186/s40623-016-0535-9.

Hu, Y., Bürgmann, R., Uchide, N., Banerjee, P., Freymueller, J.T., 2016. Stress-driven relaxation of heterogeneous upper mantle and timedependent afterslip following the 2011 Tohoku earthquake. J. Geophys. Res. 120. doi:doi:10.1002/2015JB012508.

Iinuma, T., 2018. Monitoring of the spatio-temporal change in the interplate coupling at northeastern Japan subduction zone based on the spatial gradients of surface velocity field. Geophys. J. Int. 213, 30-47.

Johnson, K.M., Mavrommatis, A., Segall, P., 2016. Small interseismic asperities and widespread aseismic creep on the northern Japan subduction interface. Geophys. Res. Lett. 43, 135-143. doi:10.1002/2015GL066707.

Kostrov, B.V., 1974. Seismic moment and energy of earthquakes and seismic flow of rock. Phys. Solid Earth 1, 23-40.

Loveless, J.P., Meade, B.J., 2010. Geodetic imaging of plate motions, slip rates, and partitioning of deformation in Japan. J. Geophys. Res. 115. doi:10.1029/2008JB006248. 
Loveless, J.P., Meade, B.J., 2016. Two decades of spatiotemporal variations in subduction zone coupling offshore Japan. Earth Planet. Sci. Lett. 436, 19-30.

Mavrommatis, A.P., Segall, P., Johnson, K.M., 2014. A decadal-scale deformation transient prior to the $2011 \mathrm{Mw} 9.0$ Tohoku-oki earthquake. Geophys. Res. Lett. 41, 4486-4494. doi:10.1002/2014GL060139.

McKenzie, D.P., 1969. The relation between fault plane solutions for earthquakes and the directions of the principal stresses. Bull. Seismol. Soc. Am. 59, 591-601.

Michael, A.J., 1984. Determination of stress from slip data; faults and folds. J. Geophys. Res. 89, 11517-11526.

Michael, A.J., 1987. Use of focal mechanisms to determine stress; a control study. J. Geophys. Res. 92, 357-368.

Nakamura, W., Uchida, N., Matsuzawa, T., 2016. Spatial distribution of the faulting types of small earthquakes around the 2011 Tohoku-oki earthquake: A comprehensive search using template events. J. Geophys. Res. 121, 2591-2607.

Obara, A., Kato, K., 2016. Connecting slow earthquakes to huge earthquakes. Science 353, 253-257.

Okada, Y., Kasahara, K., Hori, S., Obara, K., Sekiguchi, S., Fujiwara, H., Yamamoto, A., 2004. Recent progress of seismic observation networks in Japan Hi-net, F-net, K-NET and KiK-net. Earth, Planets and Space , 15-28.

Ozawa, S., Nishimura, T., Munekane, H., Suito, H., Kobayashi, T., Tobita, M., Imakiire, T., 2012. Preceding, coseismic, and postseismic slips of the 2011 Tohoku earthquake. J. Geophys. Res. 117, 10.1029/2011JB009120.

Peng, S., Gomberg, J., 2010. An integrated perspective of the continuum between earthquakes and slow-slip phenomena. Nature Geo. 3, 599-607.

Perfettini, H., Avouac, J.P., 2014. The seismic cycle in the area of the 2011 Mw9.0 Tohoku-Oki earthquake. J. Geophys. Res. 119, 4469-4515. doi:10 . $1002 / 2013$ JB010697.

Platt, J.P., Kaus, B.J.P., Becker, T.W., 2008. The San Andreas transform system and the tectonics of California: an alternative approach. Earth Planet. Sci. Lett. 274, 380-391. 
Sagiya, T., 2004. A decade of GEONET: 1994-2003 - The continuous GPS observation in Japan and its impact on earthquake studies-. Earth Planet. Space 56, 29-41.

Savage, M., Aoki, Y., Unglert, K., Ohkura, T., Umakoshi, K., Shimizu, H., Iguchi, Tameguri, T., Ohminato, T., Mori, J., 2016. Stress, strain rate and anisotropy in Kyushu, Japan. Earth Planet. Sci. Lett. 439, 129-142.

Seno, T., 1999. Syntheses of the regional stress fields of the Japanese islands. Island Arc 8, 66-79.

Siebert, L., Simkin, T., 2002-. Volcanoes of the World: an Illustrated Catalog of Holocene Volcanoes and their Eruptions. volume GVP-3 of Global Volcanism Program Digital Information Series. Smithsonian Institution. Available online at http:/ / www.volcano.si.edu/search_volcano.cfm, accessed $11 / 2017$.

Simons, M., Minson, S.E., Sladen, A., Ortega, F., Jiang, J., Owen, S.E., Meng, L., Ampuero, J.P., Wei, S., Chu, R., Helmberger, D.V., Kanamori, H., Hetland, E., Moore, A.W., Webb, F.H., 2011. The 2011 magnitude 9.0 TohokuOki earthquake: Mosaicking the megathrust from seconds to centuries. Science 332, 1421-1425.

Smith, D.E., Heaton, T.H., 2011. Models of stochastic, spatially varying stress in the crust compatible with focal-mechanism data, and how stress inversions can be biased toward the stress rate. Bull. Seismol. Soc. Am. 101, 1396-1421.

Suito, H., Nishimura, T., Tobita, M., Imakiire, T., Ozawa, S., 2011. Interplate fault slip along the Japan Trench before the occurrence of the 2011 off the Pacific coast of Tohoku Earthquake as inferred from GPS data. Earth Planets Space 63, 615-619.

Sun, T., Wang, K., Iinuma, T., Hino, R., He, J., Fujimoto, H., Kido, M., Osada, Y., Miura, S., Ohta, Y., Hu, Y., 2014. Prevalence of viscoelastic relaxation after the 2011 Tohoku-oki earthquake. Nature 514, 84-87.

Takemura, S., Shiomi, K., Kimura, T., Saito, T., 2016. Systematic difference between first-motion and waveform-inversion solutions for shallow offshore earthquakes due to a low-angle dipping slab. Earth Planet. Space 68. doi:10.1186/s40623-016-0527-9. 
Terekawa, T., Matsu'ura, M., 2010. The 3-D tectonic stress fields in and around Japan inverted from centroid moment tensor data of seismic events. Tectonics 29. doi:10.1029/2009TC002626.

Townend, J., Zoback, M.D., 2006. Stress, strain, and mountain building in central Japan. J. Geophys. Res. 111. doi:10.1029/2005 JB003759.

Twiss, R.J., Unruh, J.R., 1998. Analysis of fault slip inversions: Do they constrain stress or strain rate? J. Geophys. Res. 103, 12205-12222.

Wang, K., 2000. Stressstrain 'paradox', plate coupling, and forearc seismicity at the Cascadia and Nankai subduction zones. Tectonophys. 319, 321-338.

Wang, K., Hu, Y., He, J., 2012. Deformation cycles of subduction earthquakes in a viscoelastic Earth. Nature 484, 327-332.

Wessel, P., Smith, W.H.F., 1998. New, improved version of the Generic Mapping Tools released. Eos Trans. AGU 79, 579.

Yang, Y.R., Johnson, K.M., Chuang, R.Y., 2013. Inversion for absolute deviatoric crustal stress using focal mechanisms and coseismic stress changes: The 2011 M9 Tohoku-oki, Japan, earthquake. J. Geophys. Res. 118, 55165529. doi:10.1002/jgrb. 50389.

Yokota, Y., Koketsu, K., 2015. A very long-term transient event preceding the 2011 Tohoku earthquake. Nature Comm. 6. doi:10.1038/ ncomms 6934.

Yoshida, K., Hasegawa, A., Okada, T., Iinuma, T., Ito, Y., Asano, Y., 2012. Stress before and after the 2011 great Tohoku-oki earthquake and induced earthquakes in inland areas of eastern Japan. Geophys. Res. Lett. 39. doi:10.1029/2011GL049729. 